$\operatorname{IPPP} / 07 / 10$

$\mathrm{DCPT} / 07 / 20$

\title{
Implication of the $D^{0}$ Width Difference On CP-Violation in $D^{0}-\bar{D}^{0}$ Mixing
}

\author{
PATRICIA BALi* \\ IPPP, Department of Physics, University of Durham, Durham DH1 3LE, UK
}

\begin{abstract}
Both BaBar and Belle have found evidence for a non-zero width difference in the $D^{0}-\bar{D}^{0}$ system. Although there is no direct experimental evidence for $\mathrm{CP}$-violation in $D$ mixing (yet), we show that the measured values of the width difference $y \sim \Delta \Gamma$ already imply constraints on the CP-odd phase in $D$ mixing, which, if significantly different from zero, would be an unambiguous signal of new physics.
\end{abstract}

*Patricia.Ball@durham.ac.uk 
The highlight of this year's Moriond conference on electroweak interactions and unified theories arguably was the announcement by BaBar and Belle of experimental evidence for $D^{0}-\bar{D}^{0}$ mixing [1, 2, 3], which was quickly followed by a number of theoretical analyses [4, 5, 6, 7, 8, 9]. While Refs. [4, 7, 8, 9] focused on the constraints posed, by the experimental results, on various new-physics models, Ref. [5] presented a first analysis of the implications of these results for the fundamental parameters describing $D$ mixing. The purpose of this letter is to show that the present experimental results already imply constraints on a sizeable CP-odd phase in $D$ mixing, which could only be due to new physics (NP).

To start with, let us shortly review the theoretical formalism of $D$ mixing and the experimental results, see Refs. [10, 11] for more detailed reviews. In complete analogy to $B$ mixing, $D$ mixing in the SM is due to box diagrams with internal quarks and $W$ bosons. In contrast to $B$, though, the internal quarks are down-type. Also in contrast to $B$ mixing, the GIM mechanism is much more effective, as the contribution of the heaviest down-type quark, the $b$, comes with a relative enhancement factor $\left(m_{b}^{2}-m_{s, d}^{2}\right) /\left(m_{s}^{2}-m_{d}^{2}\right)$, but also a large CKM-suppression factor $\left|V_{u b} V_{c b}^{*}\right|^{2} /\left|V_{u s} V_{c s}^{*}\right|^{2} \sim \lambda^{8}$, which renders its contribution to $D$ mixing $\sim 1 \%$ and hence negligible. As a consequence, $D$ mixing is very sensitive to the potential intervention of NP. On the other hand, it is also rather difficult to calculate the SM "background" to $D$ mixing, as the loop-diagrams are dominated by $s$ and $d$ quarks and hence sensitive to the intervention of resonances and non-perturbative QCD. The quasi-decoupling of the 3rd quark generation also implies that $\mathrm{CP}$ violation in $D$ mixing is extremely small in the SM, and hence any observation of $\mathrm{CP}$ violation will be an unambiguous signal of new physics, independently of hadronic uncertainties.

The theoretical parameters describing $D$ mixing can be defined in complete analogy to those for $B$ mixing: the time evolution of the $D^{0}$ system is described by the Schrödinger equation

$$
i \frac{\partial}{\partial t}\left(\begin{array}{c}
D^{0}(t) \\
\bar{D}^{0}(t)
\end{array}\right)=\left(M-i \frac{\Gamma}{2}\right)\left(\begin{array}{c}
D^{0}(t) \\
\bar{D}^{0}(t)
\end{array}\right)
$$

with Hermitian matrices $M$ and $\Gamma$. The off-diagonal elements of these matrices, $M_{12}$ and $\Gamma_{12}$, describe, respectively, the dispersive and absorptive parts of $D$ mixing. The flavour-eigenstates $D^{0}=(c \bar{u}), \bar{D}^{0}=(u \bar{c})$ are related to the mass-eigenstates $D_{1,2}$ by

$$
\left|D_{1,2}\right\rangle=p\left|D^{0}\right\rangle \pm q\left|\bar{D}^{0}\right\rangle
$$

with

$$
\left(\frac{q}{p}\right)^{2}=\frac{M_{12}^{*}-\frac{i}{2} \Gamma_{12}^{*}}{M_{12}-\frac{i}{2} \Gamma_{12}}
$$

$|p|^{2}+|q|^{2}=1$ by definition.

The basic observables in $D$ mixing are the mass and lifetime difference of $D_{1,2}$, which are usually normalised to the average lifetime $\Gamma=\left(\Gamma_{1}+\Gamma_{2}\right) / 2$ :

$$
x \equiv \frac{\Delta M}{\Gamma}=\frac{M_{2}-M_{1}}{\Gamma}, \quad y \equiv \frac{\Delta \Gamma}{2 \Gamma}=\frac{\Gamma_{2}-\Gamma_{1}}{2 \Gamma} .
$$


In this letter we follow the sign convention of Ref. [5], according to which $x$ is positive by definition. The sign of $y$ then has to be determined from experiment. In addition, if there is CP-violation in the $D$ system, one also has

$$
\left|\frac{q}{p}\right| \neq 1, \quad \phi \equiv \arg \left(M_{12} / \Gamma_{12}\right) \neq 0 .
$$

While previously only bounds on $x$ and $y$ were known, both BaBar and Belle have now found evidence for non-vanishing mixing in the $D$ system. BaBar has obtained this evidence from the measurement of the doubly Cabibbo-suppressed decay $D^{0} \rightarrow K^{+} \pi^{-}$ (and its CP conjugate), yielding

$$
\begin{aligned}
y^{\prime} & =(0.97 \pm 0.44(\text { stat }) \pm 0.31(\text { syst })) \times 10^{-2}, \\
x^{\prime 2} & =(-0.022 \pm 0.030(\text { stat }) \pm 0.021(\text { syst })) \times 10^{-2},
\end{aligned}
$$

while Belle obtains

$$
y_{\mathrm{CP}}=(1.31 \pm 0.32(\text { stat }) \pm 0.25(\text { syst })) \times 10^{-2}
$$

from $D^{0} \rightarrow K^{+} K^{-}, \pi^{+} \pi^{-}$and

$$
x=(0.80 \pm 0.29 \text { (stat) } \pm 0.17 \text { (syst) }) \times 10^{-2}, \quad y=(0.33 \pm 0.24(\text { stat }) \pm 0.15(\text { syst })) \times 10^{-2}
$$

from a Dalitz-plot analysis of $D^{0} \rightarrow K_{S}^{0} \pi^{+} \pi^{-}$. Here $y_{\mathrm{CP}} \rightarrow y$ in the limit of no CP violation in $D$ mixing, while the primed quantities $x^{\prime}, y^{\prime}$ are related to $x, y$ by a rotation by a strong phase $\delta_{K \pi}$ :

$$
y^{\prime}=\cos \delta_{K \pi}-x \sin \delta_{K \pi}, \quad x^{\prime}=x \cos \delta_{K \pi}+y \sin \delta_{K \pi} .
$$

Limited experimental information on this phase has been obtainted at CLEO-c [12]:

$$
\cos \delta_{K \pi}=1.09 \pm 0.66
$$

which can be translated into $\delta_{K \pi}=(0 \pm 65)^{\circ}$. An analysis with a larger data-set is underway at CLEO-c, with an expected uncertainty of $\Delta \cos \delta_{K \pi} \approx 0.1$ in the next couple of years [13]; BES-III is expected to reach $\Delta \cos \delta_{K \pi} \approx 0.04$ after 4 years of running [14]. The experimental result (10) agrees with theoretical expectations, $\delta_{K \pi}=0$ in the $\mathrm{SU}(3)$-limit and $\left|\delta_{K \pi}\right| \lesssim 15^{\circ}$ from a calculation of the amplitudes in QCD factorisation [15]. Based on these experimental results, a preliminary HFAG-average was presented at the 2007 CERN workshop "Flavour in the Era of the LHC" [13]:

$$
x=\left(8.5_{-3.1}^{+3.2}\right) \times 10^{-3}, \quad y=\left(7.1_{-2.3}^{+2.0}\right) \times 10^{-3} .
$$

Adding errors in quadrature, this implies

$$
\frac{x}{y}=1.2 \pm 0.6
$$


The exact relations between $\Delta M, \Delta \Gamma, M_{12}$ and $\Gamma_{12}$ are given by

$$
\begin{aligned}
(\Delta M)^{2}-\frac{1}{4}(\Delta \Gamma)^{2} & =4\left|M_{12}\right|^{2}-\left|\Gamma_{12}\right|^{2}, \\
(\Delta M)(\Delta \Gamma) & =4 \operatorname{Re}\left(M_{12}^{*} \Gamma_{12}\right)=4\left|M_{12}\right|\left|\Gamma_{12}\right| \cos \phi .
\end{aligned}
$$

Eq. (13) implies $x / y>0$ for $|\phi|<\pi / 2$ and $x / y<0$ for $\pi / 2<|\phi|<3 \pi / 2$. In view of the above experimental results, we assume $|\phi|<\pi / 2$ from now on.

As for the CP-violating observables, $|q / p| \neq 1$ characterises CP-violation in mixing and can be measured for instance in flavour-specific decays $D^{0} \rightarrow f$, where $\bar{D}^{0} \rightarrow f$ is possible only via mixing. The prime example is semileptonic decays with

$$
A_{\mathrm{SL}}=\frac{\Gamma\left(D^{0} \rightarrow \ell^{-} X\right)-\Gamma\left(\bar{D}^{0} \rightarrow \ell^{+} X\right)}{\Gamma\left(D^{0} \rightarrow \ell^{-} X\right)+\Gamma\left(\bar{D}^{0} \rightarrow \ell^{+} X\right)}=\frac{|q / p|^{2}-|p / q|^{2}}{|q / p|^{2}+|p / q|^{2}} .
$$

Although the B factories may have some sensitivity to this asymmetry, its measurement is severely impaired by the fact that $D$ mixing proceeds only very slowly, resulting in a large suppression factor of the mixed vs. the unmixed rate:

$$
\frac{\Gamma\left(D^{0} \rightarrow \ell^{-} X\right)}{\Gamma\left(D^{0} \rightarrow \ell^{+} X\right)}=\frac{x^{2}+y^{2}}{2+x^{2}+y^{2}} \approx 6 \times 10^{-5} .
$$

Both in the $K$ and the $B$ system the quantity

$$
A_{M} \equiv\left|\frac{q}{p}\right|-1
$$

is very small, which however need not necessarily be the case for D's. From (3) one derives the general expression

$$
\left|\frac{q}{p}\right|^{2}=\left(\frac{4+r^{2}+4 r \sin \phi}{4+r^{2}-4 r \sin \phi}\right)^{1 / 2}
$$

with $r=\left|\Gamma_{12} / M_{12}\right|$ and the weak phase $\phi$ defined in (5). In the $B$ system, one has $r \ll 1$ (the current up-to-date numbers are $r \approx 7 \times 10^{-3}$ for $B_{d}$ and $r \approx 5 \times 10^{-3}$ for $B_{s}$ [16]), so that upon expansion in $r$

$$
\left|\frac{q}{p}\right|_{B_{d, s}}^{2}=1+\left|\frac{\Gamma_{12}}{M_{12}}\right| \sin \phi+O\left(r^{2}\right) .
$$

Note that this formula refers to the definition $\phi=\arg \left(M_{12} / \Gamma_{12}\right)$, which differs by $+\pi$ from the one used in Ref. [16], $\phi=\arg \left(-M_{12} / \Gamma_{12}\right)$. For the $K$ system, one finds $r \approx$ $|\Delta \Gamma / \Delta M| \approx 2$ from experiment, but now the phase $\phi$ turns out to be small, so that

$$
\left|\frac{q}{p}\right|_{K}^{2}=1+\frac{4 r}{4+r^{2}} \phi+O\left(\phi^{2}\right) \approx 1+\phi .
$$

In both cases, $|q / p| \approx 1$ to a very good approximation. In the $D$ system, however, there is no natural hierarchy $r \ll 1$, and of course one hopes that NP-effects induce $|\phi| \gg 0$. In 


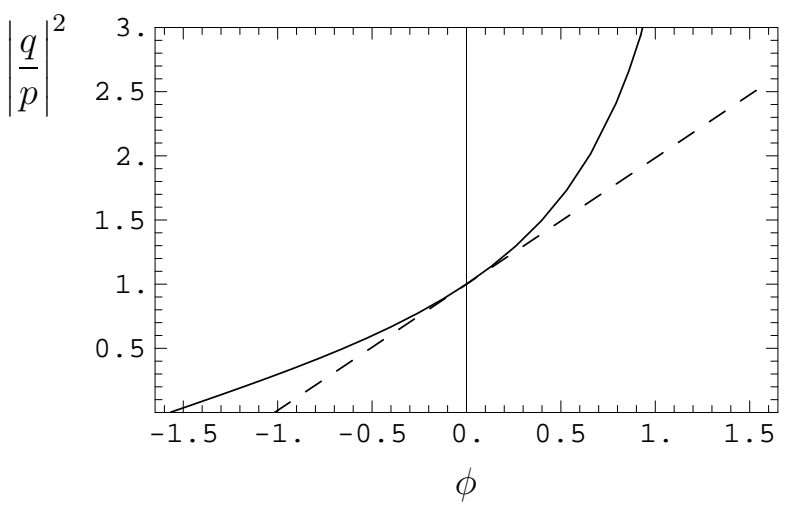

Figure 1: $|q / p|^{2}$, Eq. (20), as a function of the CP-odd phase $\phi$ for the central experimental value $\tilde{r}=7.1 / 8.5$. Solid line: full expression, dashed line: first order expansion around $\phi=0$.

this case, and because $x$ and $y$ have been measured, while $\left|M_{12}\right|$ and $\left|\Gamma_{12}\right|$ are difficult to calculate, it is convenient to express $|q / p|_{D}$ in terms of $x, y, \phi$, using the exact relations (13). From (3), and defining $\tilde{r}=y / x$, we then obtain

$$
\begin{aligned}
\left|\frac{q}{p}\right|^{2}=\frac{1}{\sqrt{2}\left(1+\tilde{r}^{2}\right)} & \left\{2\left(1+\tilde{r}^{2}\right)^{2}+16 \tilde{r}^{2} \tan ^{2} \phi\right. \\
& \left.+8 \tilde{r} \tan \phi \sec \phi \sqrt{\left(1+\tilde{r}^{2}\right)^{2}-\left(1-\tilde{r}^{2}\right)^{2} \sin ^{2} \phi}\right\}^{1 / 2} .
\end{aligned}
$$

Note that for finite $x y$ and $\phi= \pm \pi / 2,|q / p|$ diverges because $x y \rightarrow 0$ for $\phi \rightarrow \pm \pi / 2$ from (13). In Fig. 1 we plot $|q / p|^{2}$ as function of $\phi$, for the central experimental value from HFAG, $\tilde{r}=7.1 / 8.5$, Eq. (11). It is obvious that even for moderate values of $\phi$ the small- $\phi$ expansion is not really reliable.

What is the currently available experimental information on CP-violating in $D$ mixing, i.e. $|q / p|$ and $\phi$ ? As already mentioned, the semileptonic CP-asymmetry (14) has not been measured yet. What has been measured, though, is the effect of CP-violation on the time-dependent rates of $D^{0} \rightarrow K^{+} \pi^{-}$and $\bar{D}^{0} \rightarrow K^{-} \pi^{+}$. The BaBar collaboration has parametrised these rates as

$$
\begin{aligned}
& \Gamma\left(D^{0}(t) \rightarrow K^{+} \pi^{-}\right) \propto e^{-\Gamma t}\left[R_{D}+\sqrt{R_{D}} y_{+}^{\prime} \Gamma t+\frac{x_{+}^{\prime 2}+y_{+}^{\prime 2}}{4}(\Gamma t)^{2}\right], \\
& \Gamma\left(\bar{D}^{0}(t) \rightarrow K^{-} \pi^{+}\right) \propto e^{-\Gamma t}\left[R_{D}+\sqrt{R_{D}} y_{-}^{\prime} \Gamma t+\frac{x_{-}^{\prime 2}+y_{-}^{\prime 2}}{4}(\Gamma t)^{2}\right]
\end{aligned}
$$

and fit the $D^{0}$ and $\bar{D}^{0}$ samples separately. They find [2]

$$
\begin{aligned}
& y_{+}^{\prime}=(9.8 \pm 6.4(\text { stat }) \pm 4.5(\text { syst })) \times 10^{-3}, \\
& y_{-}^{\prime}=(9.6 \pm 6.1(\text { stat }) \pm 4.3(\text { syst })) \times 10^{-3} .
\end{aligned}
$$

Adding errors in quadrature, this means $y_{+}^{\prime} / y_{-}^{\prime}=1.0 \pm 1.1$. BaBar also obtains values for $x_{ \pm}^{\prime 2}$ which we do not quote here, because the sensitivity to the quadratic term in (21) is 

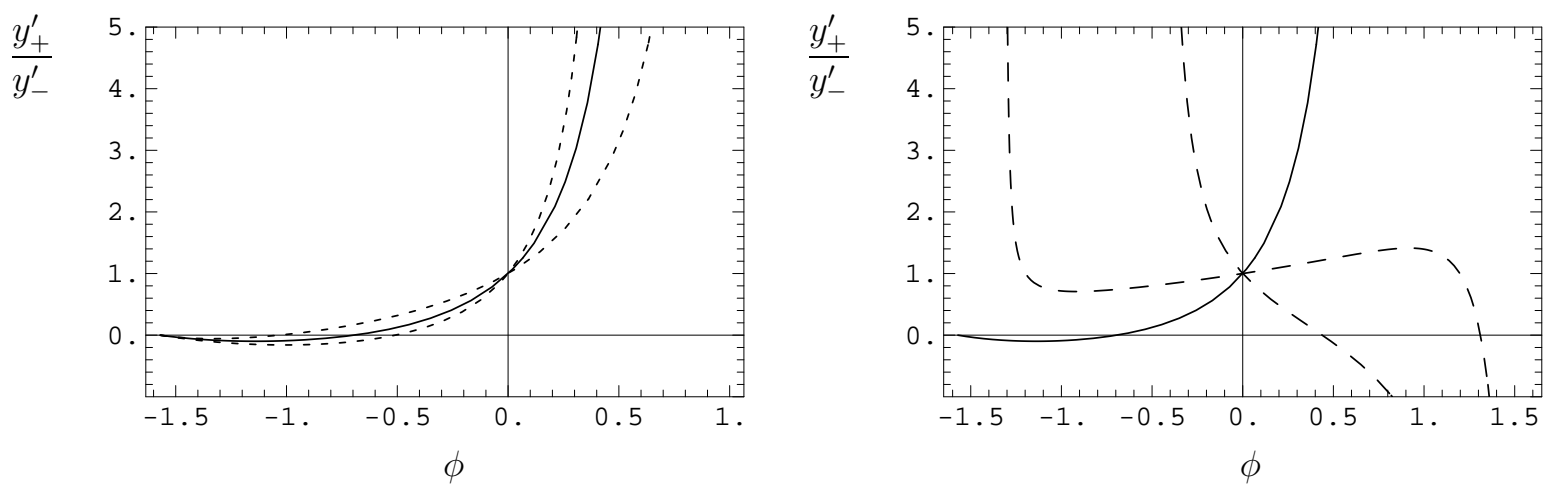

Figure 2: Left: $y_{+}^{\prime} / y_{-}^{\prime}$ as function of $\phi$ for $x / y=1.2$ (solid line) and $x / y=\{0.6,1.8\}$ (dashed lines), from Eq. (11). $\delta_{K \pi}=0$. Right: $y_{+}^{\prime} / y_{-}^{\prime}$ as function of $\phi$ for $x / y=1.2$ for $\delta_{K \pi}=0$ (solid line) and $\delta_{K \pi}= \pm 65^{\circ}$ (dashed lines).

less than that to the linear term in $y_{ \pm}^{\prime} \cdot R_{D}^{1 / 2}$ is the ratio of the doubly Cabbibo-suppressed to the Cabibbo-favoured amplitude, $R_{D}^{1 / 2}=\left|A\left(D^{0} \rightarrow K^{+} \pi^{-}\right) / A\left(D^{0} \rightarrow K^{-} \pi^{+}\right)\right|$. $\delta_{K \pi}$ is the relative strong phase in the Cabibbo-favoured and suppressed amplitudes:

$$
\frac{A\left(D^{0} \rightarrow K^{+} \pi^{-}\right)}{A\left(\bar{D}^{0} \rightarrow K^{+} \pi^{-}\right)}=-\sqrt{R_{D}} e^{-i \delta_{K \pi}}
$$

the minus-sign comes from the relative sign between the CKM matrix elements $V_{c d}$ and $V_{u s}$. In the limit of no CP-violation in the decay amplitude, one has $\left|A\left(D^{0} \rightarrow K^{-} \pi^{+}\right)\right|=$ $\left|A\left(\bar{D}^{0} \rightarrow K^{+} \pi^{-}\right)\right|$, which is expected to be a very good approximation, in view of the fact that the decay is solely due to a tree-level amplitude. Then the relation of $y_{ \pm}^{\prime}$ to $x, y$ and $\phi$ is given by

$$
\begin{aligned}
& y_{+}^{\prime}=\left|\frac{q}{p}\right|\left\{\left(y \cos \delta_{K \pi}-x \sin \delta_{K \pi}\right) \cos \phi+\left(x \cos \delta_{K \pi}+y \sin \delta_{K \pi}\right) \sin \phi\right\}, \\
& y_{-}^{\prime}=\left|\frac{p}{q}\right|\left\{\left(y \cos \delta_{K \pi}-x \sin \delta_{K \pi}\right) \cos \phi-\left(x \cos \delta_{K \pi}+y \sin \delta_{K \pi}\right) \sin \phi\right\} .
\end{aligned}
$$

Presently, the experimental result for $y_{+}^{\prime} / y_{-}^{\prime}$ is compatible with 1 , although with considerable uncertainties. Any significant deviation from 1 would be a sign for new physics. In Fig. 2 we plot $y_{+}^{\prime} / y_{-}^{\prime}$ as function of $\phi$, for different values of $x / y$ and $\delta_{K \pi}$. The figures clearly show that the value of $y_{+}^{\prime} / y_{-}^{\prime}$ is very sensitive to the phase $\phi$, at least if $\delta_{K \pi}$ is not too close to $-65^{\circ}$, which corresponds to the nearly constant dashed line in Fig. $2 \mathrm{~b}$. The reason for this dependence on $\delta_{K \pi}$ becomes clearer if $y_{+}^{\prime} / y_{-}^{\prime}$ is expanded to first order in $\phi$ :

$$
\frac{y_{+}^{\prime}}{y_{-}}=1-2 \phi \frac{x\left(x^{2}+2 y^{2}\right) \cos \delta_{K \pi}+y^{3} \sin \delta_{K \pi}}{\left(x^{2}+y^{2}\right)\left(x \sin \delta_{K \pi}-y \cos \delta_{K \pi}\right)}+O\left(\phi^{2}\right) .
$$

For the central values of $x$ and $y$, Eq. (11), this amounts to $1+3.4 \phi$ for $\delta_{K \pi}=0,1-3.3 \phi$ for $\delta_{K \pi}=+65^{\circ}$ and $1+0.45 \phi$ for $\delta_{K \pi}=-65^{\circ}$, which explains the shape of the curves in Fig. 2b. Evidently it is important to reduce the uncertainty of $\delta_{K \pi}$, which, as mentioned 


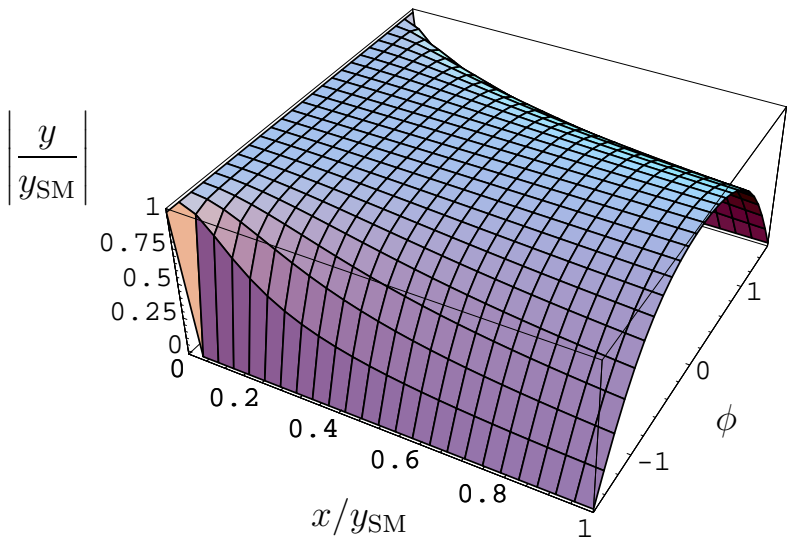

Figure 3: Plot of $\left|\Delta \Gamma / \Delta \Gamma^{\mathrm{SM}}\right|$, Eq. (26) , as a function of $x / y_{\mathrm{SM}}$ and $\phi$.

earlier, will be achieved within the next few years. On the other hand, as shown in Fig. 2 a,$y_{+}^{\prime} / y_{-}^{\prime}$, which depends only on the ratio $x / y$, but not $x$ and $y$ separately, is not very sensitive to the precise value of that ratio, but very much so to $\phi$. The conclusion is that, even if $x / y$ itself cannot be determined very precisely, $y_{+}^{\prime} / y_{-}^{\prime}$ will nonetheless be a powerful tool to constrain $\phi$, at least once $\delta_{K \pi}$ will be known more precisely. Already now very large values $\phi \sim \pi / 2$ are excluded.

Another, more theory-dependent constraint on $\phi$ can be derived from the value of $y$. This argument centers around the fact that (a) the experimental result (11) is at the top end of theoretical predictions $y_{\mathrm{SM}} \sim 1 \%$ [17] and (b) new physics indicated by a non-zero value of $\phi$ always reduces the lifetime difference, independently of the value of $x$. This observation is similar to what was found, some time ago, for the $B_{s}$ system [18]. In order to derive it, we assume that new physics does not affect $\Gamma_{12} 11$ so that $\Gamma_{12}=\Gamma_{12}^{\mathrm{SM}}$. We then have $2\left|\Gamma_{12}\right|=\Delta \Gamma^{\mathrm{SM}}$ and hence $\left|y_{\mathrm{SM}}\right|=\left|\Gamma_{12}\right| / \Gamma$. Using the relations (13), we can then express the ratio $\left|\Delta \Gamma / \Delta \Gamma^{\mathrm{SM}}\right|$ in terms of $y_{\mathrm{SM}}, x$ and $\phi$ :

$$
\left|\frac{y}{y_{\mathrm{SM}}}\right|=\left|\frac{\Delta \Gamma}{\Delta \Gamma^{\mathrm{SM}}}\right|=\left(\frac{y_{\mathrm{SM}}^{2}+x^{2}}{y_{\mathrm{SM}}^{2}+x^{2} / \cos ^{2} \phi}\right)^{1 / 2} .
$$

This implies that new physics always reduces the lifetime difference, independently of the value of $x$ (and any new physics in the mass difference). In particular one has $y=0$ for $\phi= \pm \pi / 2$ and $x \neq 0$, which follows from the 2 nd relation (13). Eq. (26) is the manifestation of the fact that one does not need to observe CP-violation in order to constrain it. A famous example for this is the unitarity triangle in $B$ physics, whose sides are determined from CP-conserving quantities only, but nonetheless allow a precise measurement of the size of CP-violation in the SM, via the angles and the area of the triangle. In Fig. 3, we plot $\left|\Delta \Gamma / \Delta \Gamma^{\mathrm{SM}}\right|$ as a function of $r=x / y_{\mathrm{SM}}$. The zero at $\phi= \pm \pi / 2$ is clearly visible. The experimental value $\left|y / y_{\mathrm{SM}}\right|=O(1)$ then excludes phases $\phi$ close to $\pm \pi / 2$. In order to make more quantitative statements, apparently a more precise calculation of $y_{\mathrm{SM}}$ is needed.

\footnotetext{
${ }^{1}$ See, however, Ref. [19] for a discussion of the effect of tiny NP admixtures to $\Gamma_{12}$.
} 

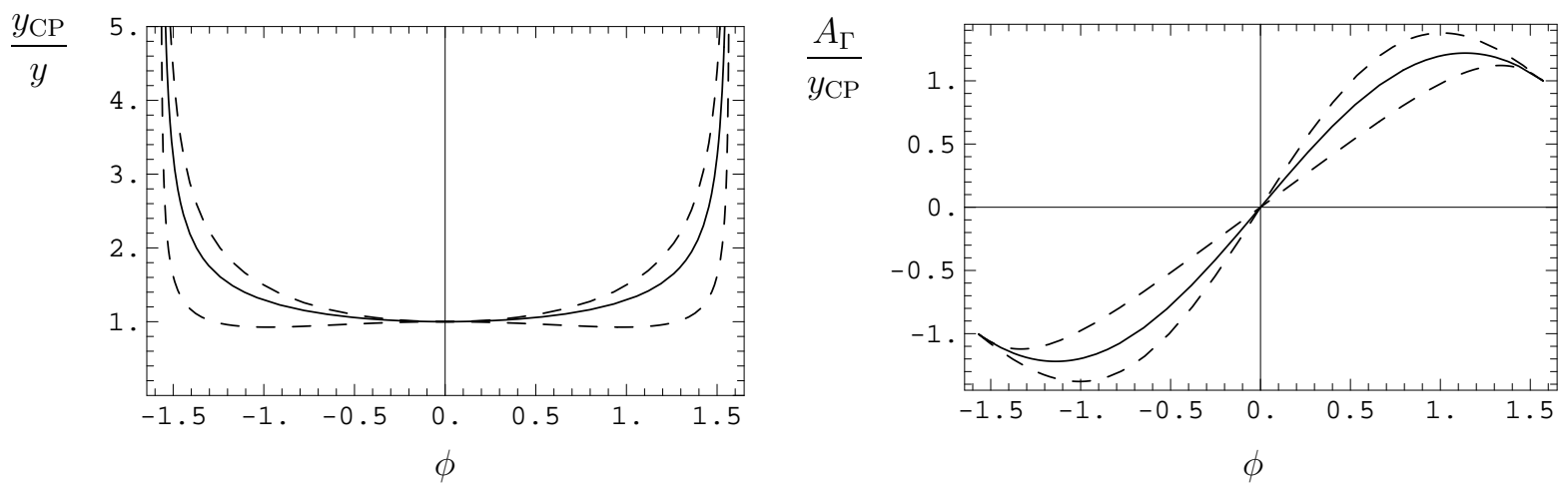

Figure 4: Left: $y_{\mathrm{CP}} / y$ as function of $\phi$, for $x / y=1.2$ (solid line) and $x / y=\{0.6,1.8\}$ (dashed lines), see Eq. (12). Right: $A_{\Gamma} / y_{\mathrm{CP}}$ as function of $\phi$.

Two more CP-sensitive observables related to $D^{0} \rightarrow K^{+} K^{-}$have been measured by the Belle collaboration [3]:

$$
\begin{aligned}
y_{\mathrm{CP}} & =\frac{1}{2 \Gamma}\left[\Gamma\left(D^{0} \rightarrow K^{+} K^{-}\right)+\Gamma\left(\bar{D}^{0} \rightarrow K^{+} K^{-}\right)\right]-1 \\
& =\frac{1}{2}\left(\left|\frac{q}{p}\right|+\left|\frac{p}{q}\right|\right) y \cos \phi+\frac{1}{2}\left(\left|\frac{q}{p}\right|-\left|\frac{p}{q}\right|\right) x \sin \phi, \\
A_{\Gamma} & =\frac{1}{2 \Gamma}\left[\Gamma\left(D^{0} \rightarrow K^{+} K^{-}\right)-\Gamma\left(\bar{D}^{0} \rightarrow K^{+} K^{-}\right)\right]-1 \\
& =\frac{1}{2}\left(\left|\frac{q}{p}\right|-\left|\frac{p}{q}\right|\right) y \cos \phi+\frac{1}{2}\left(\left|\frac{q}{p}\right|+\left|\frac{p}{q}\right|\right) x \sin \phi .
\end{aligned}
$$

The present experimental value of $y_{\mathrm{CP}}$ is given in (77), that for $A_{\Gamma}$ is $(0.01 \pm 0.30$ (stat) \pm 0.15 (syst) $) \times 10^{-2}$. Again, we can study the dependence of these observables on $\phi$. In Fig. 4 a we plot the ratio $y_{\mathrm{CP}} / y$, which is a function of $x / y$ and $\phi$, in dependence on $\phi$. As it turns out, this quantity is far less sensitive to $\phi$ than $y_{+}^{\prime} / y_{-}^{\prime}$, the reason being that its deviation from 1 is only a second-order effect in $\phi$ :

$$
y_{\mathrm{CP}}=y\left\{1+\phi^{2} \frac{x^{4}+x^{2} y^{2}-y^{4}}{2\left(x^{2}+y^{2}\right)^{2}}+O\left(\phi^{4}\right)\right\} .
$$

Hence, unless the experimental accuracy is dramatically increased, and because the results on $y_{+}^{\prime} / y_{-}^{\prime}$ and $y / y_{\text {SM }}$ already exclude a large CP-odd phase $\phi \approx \pm \pi / 2$, it is safe to interpret $y_{\mathrm{CP}}$ as measurement of $y$. In Fig. $4 \mathrm{~b}$ we plot the quantity $A_{\Gamma} / y_{\mathrm{CP}}$. Also here there is a distinctive dependence on $\phi$, with $A_{\Gamma} / y \propto \phi$ for small $\phi$, but the effect is less dramatic than that in $y_{+}^{\prime} / y_{-}^{\prime}$.

In conclusion, we find that the experimental results on $D$ mixing reported by BaBar and Belle already exclude extreme values of the CP-odd phase $\phi$ close to $\pm \pi / 2$. This follows from the result for $y$, which is close to the top end of theoretical predictions and can only be reduced by new physics, and from $y_{+}^{\prime} / y_{-}^{\prime} \sim 1$. While $y_{+}^{\prime} / y_{-}^{\prime}-1$ vanishes in the limit of no $\mathrm{CP}$-violation, $y \sim \Delta \Gamma$ is a $\mathrm{CP}$-conserving observable, which demonstrates 
the usefulness of such quantities in constraining CP-odd phases. Also $y_{\mathrm{CP}}, A_{\Gamma}$ and the ratio $A_{\Gamma} / y_{\mathrm{CP}}$ can be useful in constraining $\phi$. As long as there is no major breakthrough in theoretical predictions for $D$ mixing, which are held back by the fact that the $D$ meson is at the same time too heavy and too light for current theoretical tools to get a proper grip on the problem, the long-distance SM contributions to $x$ will completely obscure any NP contributions and their detection. The observation of CP violation, however, presents a theoretically clean way for NP to manifest itself and it is to be hoped that in the near future, i.e. at the $B$ factories or the $\mathrm{LHC}$, at least one of the plentiful opportunities for NP to show up in CP violation [20] will be realised.

\section{Acknowledgments}

This work was supported in part by the EU networks contract Nos. MRTN-CT-2006035482, Flavianet, and MRTN-CT-2006-035505, Heptools.

\section{References}

[1] M. Staric (Belle), talk given at 42nd Rencontres de Moriond, Electroweak Interactions and Unified Theories, La Thuile, Italy, March 2007;

K. Flood (BaBar), talk given at the same conference.

[2] B. Aubert et al. [BABAR Collaboration], arXiv:hep-ex/0703020.

[3] K. Abe [Belle Collaboration], arXiv:hep-ex/0703036.

[4] M. Ciuchini et al., arXiv:hep-ph/0703204.

[5] Y. Nir, arXiv:hep-ph/0703235.

[6] P. Ball, arXiv:hep-ph/0703245.

[7] M. Blanke et al., arXiv:hep-ph/0703254.

[8] X. G. He and G. Valencia, arXiv:hep-ph/0703270.

[9] C. H. Chen, C. Q. Geng and T. C. Yuan, arXiv:0704.0601.

[10] G. Burdman and I. Shipsey, Ann. Rev. Nucl. Part. Sci. 53, 431 (2003) arXiv:hep-ph/0310076.

[11] D. Asner, review on D mixing in W. M. Yao et al. [Particle Data Group], J. Phys. G 33 (2006) 1;

I. Shipsey, Int. J. Mod. Phys. A 21 (2006) 5381 arXiv:hep-ex/0607070;

A. A. Petrov, Int. J. Mod. Phys. A 21 (2006) 5686 arXiv:hep-ph/0611361. 
[12] W. M. Sun [CLEO Collaboration], AIP Conf. Proc. 842 (2006) 693 arXiv:hep-ex/0603031;

D. Asner et al. [CLEO Collaboration], Int. J. Mod. Phys. A 21 (2006) 5456 arXiv:hep-ex/0607078].

[13] D. Asner, talk given at workshop Flavour Physics in the Era of the LHC, CERN, March 07, http://mlm.home.cern.ch/mlm/FlavLHC.html.

[14] X. D. Cheng et al., arXiviarXiv:0704.0120.

[15] D. N. Gao, Phys. Lett. B 645 (2007) 59 arXiv:hep-ph/0610389.

[16] A. Lenz and U. Nierste, arXiv:hep-ph/0612167.

[17] H. Georgi, Phys. Lett. B 297, 353 (1992) [arXiv:hep-ph/9209291];

T. Ohl, G. Ricciardi and E. H. Simmons, Nucl. Phys. B 403, 605 (1993) arXiv:hep-ph/9301212];

I. I. Y. Bigi and N. G. Uraltsev, Nucl. Phys. B 592, 92 (2001) arXiv:hep-ph/0005089];

A. F. Falk, Y. Grossman, Z. Ligeti and A. A. Petrov, Phys. Rev. D 65, 054034 (2002) arXiv:hep-ph/0110317;

A. F. Falk et al., Phys. Rev. D 69, 114021 (2004) arXiv:hep-ph/0402204.

[18] Y. Grossman, Phys. Lett. B 380 (1996) 99 arXiv:hep-ph/9603244].

[19] E. Golowich, S. Pakvasa and A. A. Petrov, arXiv:hep-ph/0610039.

[20] P. Ball, J. M. Frere and J. Matias, Nucl. Phys. B 572 (2000) 3 arXiv:hep-ph/9910211;

P. Ball and R. Zwicky, JHEP 0604 (2006) 046 [arXiv:hep-ph/0603232];

P. Ball and R. Fleischer, Eur. Phys. J. C 48, 413 (2006) [arXiv:hep-ph/0604249];

P. Ball and R. Zwicky, Phys. Lett. B 642 (2006) 478 [arXiv:hep-ph/0609037];

P. Ball, G. W. Jones and R. Zwicky, Phys. Rev. D 75 (2007) 054004 arXiv:hep-ph/0612081]. 